\title{
The importance of memory and executive function in aphasia: evidence from the treatment of anomia using errorless and errorful learning.
}

Link to publication record in Manchester Research Explorer

Citation for published version (APA):

Lambon Ralph, M., Lambon, R. MA., Fillingham, JK., Meyer, A. (Ed.), Wheeldon, L. R. (Ed.), \& Krott, A. (Ed.) (2007). The importance of memory and executive function in aphasia: evidence from the treatment of anomia using errorless and errorful learning. In Automaticity and control in language processing (pp. 193-216). Psychology Press Ltd.

\section{Published in:}

Automaticity and control in language processing

\section{Citing this paper}

Please note that where the full-text provided on Manchester Research Explorer is the Author Accepted Manuscript or Proof version this may differ from the final Published version. If citing, it is advised that you check and use the publisher's definitive version.

\section{General rights}

Copyright and moral rights for the publications made accessible in the Research Explorer are retained by the authors and/or other copyright owners and it is a condition of accessing publications that users recognise and abide by the legal requirements associated with these rights.

\section{Takedown policy}

If you believe that this document breaches copyright please refer to the University of Manchester's Takedown Procedures [http://man.ac.uk/04Y6Bo] or contact uml.scholarlycommunications@manchester.ac.uk providing relevant details, so we can investigate your claim.

\section{OPEN ACCESS}




\title{
9 The importance of memory and executive function in aphasia: Evidence from the treatment of anomia using errorless and errorful learning
}

\author{
Matthew A. Lambon Ralph and \\ Joanne K. Fillingham \\ University of Manchester, UK
}

\section{Introduction}

Neural plasticity, that is, the ability of the brain to change its function, is a current and fundamental issue in neuroscience (e.g., Buonomano \& Merzenich, 1998; Tallal, Merzenich, Miller, \& Jenkins, 1998). Work at this level of basic science has shown that the mature brain is, in principle, capable of "rewiring" itself so that new functions can be learnt by brain areas that previously performed other processes (Buonomano \& Merzenich, 1998). This basic science has been extended to the applied level (e.g., Tallal et al., 1998; Wilson \& Evans, 1996). This applied research suggests that a particular form of therapy/remediation programme, known as errorless learning, might have advantages over more traditional trial-and-error methods. The basic premise behind errorless learning is that learning/recovery may be limited by patients' errors in that, not only may they correctly reinforce the link between stimulus and correct responses but they might also reinforce the association with erroneous responses. By adjusting the intervention such that the patients are much less likely to make errors, better learning arises because the patients reinforce only the correct response. In turn, computational neuroscience is beginning to provide a link between clinical application of errorless learning and basic neuroscience. This work has shown that the functioning of neuron-like processing units does not alter if the system reinforces its own errors, but change can follow in circumstances like errorless learning (McClelland, Thomas, McCandliss, \& Fiez, 1999).

Errorless learning has a long history. It was first studied and proved effective in the area of animal learning. Under standard procedures, pigeons demonstrate very poor learning of red-green visual discrimination even after 
repeated exposures. Terrace (1963) found that if an errorless learning method was adopted, pigeons' learning of the same discrimination was very much better. Since then, it has been used as a rehabilitation method in a variety of areas. It has been used as a behaviour-modification technique with children and adults (Brownjohn, 1988; Cipani \& Spooner, 1997; Ducharme, 1996; Ducharme, Atkinson, \& Poulton, 2000). These methods have been used to teach auditory discrimination skills to children with language-based learning impairments and to children and adults with Down's syndrome (Brownjohn, 1988; Duffy \& Wishart, 1987, 1994; McCandliss, Fiez, Protopapas, Conway, \& McClelland, 2002; Merzenich, Jenkins, Johnston, Schreiner, Miller, \& Tallal, 1996; Tallal et al., 1998). It has been used with people with psychiatric illnesses to teach a variety of tasks, including naming (Kern, 1996; O'Carroll, Russell, Lawrie, \& Johnstone, 1999; Wykes, Reeder, Corner, Williams, \& Everitt, 1999). The occupational therapy literature reports it as a technique for dyspraxia intervention (Jackson, 1999). More recently, studies have found that errorless training methods are more successful when teaching golf putting to normal adults (Maxwell, Masters, Kerr, \& Weedon, 2001).

Baddeley (1992) suggested that errorless learning was a possible principle for the practice of memory rehabilitation, and it is in the domain of amnesia that much of the recent literature has been focused. Wilson and colleagues have studied the use of errorless learning extensively and compared this with errorful methods. With this technique they have taught people with amnesia and memory impairment to do a variety of tasks, including learning names of objects and people, programming an electronic aid, remembering orientation items, and learning items of general knowledge (Baddeley \& Wilson, 1994; Clare, Wilson, Breen, \& Hodges, 1999; Clare, Wilson, Carter, Breen, Gosses, \& Hodges, 2000; Evans et al., 2000; Wilson \& Evans, 1996; Wilson, Baddeley, Evans, \& Shiel, 1994). Squires, Hunkin, Parkin, and colleagues have also examined errorless and errorful learning. They taught people with amnesia word associations, names of objects and people, and basic commands and procedures associated with use of a word-processing package (Hunkin, Squires, Parkin, \& Tidy, 1998; Hunkin, Squires, Aldrich, \& Parkin, 1998; Parkin, Hunkin, \& Squires, 1998; Squires, Hunkin, \& Parkin, 1997).

Wilson and colleagues pioneered the use of errorless learning for the treatment of memory impairment across a range of people (Clare et al., 1999; Wilson et al., 1994). The existing literature provides many demonstrations that amnesic subjects are better able to learn new and relearn old information by errorless techniques. The errorless method has been successfully applied to a variety of learning situations, including relearning proper nouns and object names. These studies can be split into two types: experimental and therapeutic. In the experimental studies, Wilson and colleagues have directly compared errorless and errorful learning. They have asked people with amnesia to learn arbitrary associations (paired associate learning and stem completion) and have found that patients consistently do better if 
the learning paradigm prevents patients from making errorful responses. For example, Wilson et al. (1994) taught PS, a patient with dense amnesia following a thalamic stroke, the names of fictitious people by errorful and errorless techniques. Ten first names and 10 surnames were trained with each technique. Three successive learning trials were given for each technique followed by nine test trails, spread over a 30-minute period. In the errorful condition, PS was given the first letter of the name and was asked to guess. If, after three guesses, he was still incorrect, the correct name was given, and he was asked to write it down. In the errorless condition, PS was told the first letter, and then the name and was asked to write it down. Wilson et al. (1994) found that in eight of the nine test trials, errorless learning resulted in superior learning. In the therapeutic studies, Wilson and colleagues have extended these experimental findings and have shown that people with amnesia can learn to use memory aids, learn new tasks, or relearn the names of objects or people by the use of errorless learning. For example, Clare et al. (1999) retaught VJ, a man in the early stages of Alzheimer's disease, the names of 11 members of his social club by an errorless technique. The therapy comprised the following elements: learning a mnemonic for each person; completing the name with a step-by-step reduction of the letters provided (vanishing cues); writing the name by filling in the gaps; recalling the mnemonic used; repeating the name; and, finally, recall at short but gradually increasing intervals. The therapy consisted of 21 sessions twice weekly. VJ also practised the same procedure with family members at home three times a day, and he was encouraged to name the members while he was at his social club. VJ was tested on the 11 names after each therapy session. These continual assessments showed that the proportion of faces correctly named increased from $22 \%$ at baseline to $98 \%$ after training. Additional testing found that this benefit was maintained after 9 months.

If we consider results from beyond the amnesia literature, however, it is clear that errorless learning is advantageous for people other than just those with impaired memory. For a complete account, other aspects of the processes that underpin learning (and relearning) need to be considered. For example, it can be very difficult for adults to learn to perceive certain speech contrasts that their native language does not use. Native Japanese people have persistent difficulty in distinguishing the English liquids [r] and [1]. Even after years of exposure to English, Japanese adults are still markedly poorer at discriminating between these sounds than native English speakers. Recent studies (see below) have been able to remediate these persistent perceptual difficulties by errorless learning. Such demonstrations are important because these participants are entirely neurologically normal, and yet errorless learning can induce plasticity in their mature language system.

McClelland et al. (1999) used a computational model to test the hypothesis that the Japanese adults' poor learning was due to a general learning mechanism that reinforces perceptual categories learnt early in life but prevents new learning. More specifically, Hebbian learning might maintain 
pre-established perceptual representations even in the face of new experience. McClelland et al. (1999) trained computational, Kohonen networks in English-like and Japanese-like environments. In the English version, the model was trained to discriminate between six different abstract "phonological" representations, including $/ 1 /$ and $/ \mathrm{r} /$. Tokens of these two phonemes were sampled from two similar but somewhat different distributions. The Japanese version was identical except that the distributions of $/ 1 /$ and $/ \mathrm{r} /$ were identical to mirror the fact that there is no distinction between these two phonemes in the real Japanese language. After training, the representational layer of the English model had been structured to reflect six different phonemes, including separate spaces for $/ 1 /$ and $/ \mathrm{r} /$. Unsurprisingly, the representational space in the Japanese model contained only five distinctions with /1/ and $/ \mathrm{r} /$ occupying the same part. In the next, critical step, McClelland et al. (1999) switched the Japanese model to the English-like environment in which tokens of $/ 1 /$ and $/ \mathrm{r} /$ were drawn from overlapping but nonidentical distributions. Despite this switch, the model, like Japanese people, failed to learn the $/ 1 /-/ r /$ discrimination. This followed because both $/ 1 /$ and $/ \mathrm{r} /$ inputs were captured by the same, single phonological representation, and this incorrect association was continually reinforced by the Hebbian learning used in this model. Although the model, like Japanese people, appeared to be perpetually stuck, McClelland et al. (1999) were able to demonstrate that plasticity and effective learning were possible, given the right circumstances. Instead of presenting the model with the standard English-like environment, McClelland et al. (1999) included exaggerated tokens of $/ 1 /$ and $/ \mathrm{r} /$, thus increasing the separation of their distributions. In these circumstances, the model no longer collapsed / $/ /$ and $/ \mathrm{r} /$ into the same single representation but altered the representational layer to include six separate phonemes. Most importantly, when the model was returned to the English-like environment with overlapping / $/ 1 /$ and $/ \mathrm{r} /$ distributions, it was able to accurately discriminate between the two phonemes.

These simulations provide the obvious prediction that the use of exaggerated contrasts might prove effective for human subjects. Previous work suggests that this type of training regime can be useful for children with language-learning impairments (Merzenich et al., 1996; Tallal et al., 1998). McCandliss, et al. (2002) conducted an empirical study with Japanese adults in order to test the prediction of the simulations more directly. This study contrasted two important factors: errorless versus errorful and with feedback versus without feedback. The errorless condition used an adaptive training method. Japanese subjects began with exaggerated tokens of /1/ and $/ \mathrm{r} /$ embedded within a simple word frame (lock and rock). A staircase method was used to move the tokens gradually toward those found in normal English. Closer tokens of $/ 1 /$ and $/ r /$ were used once the subject had made eight successive correct discriminations, while more disparate examples were used if the subject made only one error. This asymmetric staircase method provided an "error-reduction" intervention in which errors were deliberately controlled 
and minimized. In contrast, in the errorful condition, the Japanese subjects were presented with standard tokens of $/ 1 /$ and $/ \mathrm{r} /$ throughout the training sessions. These two training regimes were crossed with the feedback factor. Half the subjects were told whether their discrimination choice was correct while the remainder received no feedback. In the no-feedback condition, the prediction of the computational model was upheld. The adaptive, errorreduction method produced significant improvement in $/ 1 /-/ \mathrm{r} /$ discrimination, while the errorful method produced no benefit relative to control. McCandliss et al. (2002) also found that feedback was a critical factor: subjects in the errorful condition who received feedback about their discriminations learned as well as those people in the adaptive, error-reducing condition.

When this study is taken together with those described above, it is clear that there is a wide range of learning situations and of participants/patients for which errorless learning is beneficial. In addition, the McCandliss et al. (2002) study demonstrates that some subjects are able to use feedback to control for the inherent problems associated with learning in an errorful situation. While amnesia may be an important factor when choosing between an errorful or errorless intervention (Baddeley \& Wilson, 1994), memory impairments do not provide a complete explanation for the whole literature: there are a range of situations in which neurologically intact subjects benefit from an errorless technique (Maxwell et al., 2001; McCandliss et al., 2002). In addition, there are certain circumstances in which even normal subjects with feedback available still perform better with an errorless method (e.g., when learning a complex motor skill; Maxwell et al., 2001).

\section{Review of previous therapy studies for treating aphasic word-finding difficulties}

The amnesia studies raise the possibility that errorless learning might be a technique that could be used to treat aphasic word-finding difficulties (anomia) and, perhaps, a wide variety of acquired language deficits. Studies of children with developmental disorders and normal Japanese adults (McCandliss et al., 2002; Tallal et al., 1998) also indicate that errorless learning methods can be successfully extended from the memory to the language domain. As far as we are aware, the aphasia treatment literature contains no studies that have directly investigated the use of errorless learning. A closer look at the literature, however, highlights a number of studies that are comparable to the errorless method. For example, Morris, Franklin, Ellis, Turner, \& Bailey (1996) conducted a study designed to treat word deafness that is analogous to other reports in the errorless learning literature (McCandliss et al., 2002; Tallal et al., 1998). Morris et al.'s (1996) investigation highlights the fact that although the aphasic therapy literature contains no studies that have set out to examine errorless learning specifically, it does include interventions that either reduce or eliminate errors. Given that the amnesia literature contains many examples of therapies aimed at treating word-finding 
problems, an obvious question is whether errorless techniques might also prove effective for treating anomia in people with aphasia. This would be especially interesting given that word-finding difficulties are perhaps one of the most common and disabling aphasic symptoms (Lambon Ralph, Moriarty, Sage, \& Group, 2002).

We undertook, therefore, a review of the anomia treatment literature, starting from the seminal paper by Howard, Patterson, Franklin, Orchard Lisle, \& Morton (1985). Studies were categorized into one of three classifications: error elimination (patient errors are completely eliminated from the therapy), error reduction (the therapy is designed to reduce or minimize patient errors), and errorful learning (no control is exerted over patient errors). The relative merits of each approach were considered in terms of three efficacy measures - improvement immediately after therapy, residual benefit after a period of no therapy (follow-up testing), and generalization to untreated items. Studies were then split by aphasia type (fluent versus nonfluent aphasia), principal impairment (expressive versus both receptive and expressive aphasia), and therapy method (receptive or expressive therapy) to test for variation in outcome for these important factors.

It is becoming increasingly recognized that cognition is an important factor in determining recovery outcome (Helm-Estabrooks, 2002). Robertson and Murre (1999) argued that not only are age and education determinants of recovery, but there is now evidence that attentional control and level of awareness are also predictors. These cognitive factors may, in turn, reflect the integrity of frontal lobe function. The aphasia and amnesia literatures are such that it is difficult to make comparisons and theoretical predictions about the role of cognitive processes in language recovery/rehabilitation. This is because aphasia studies rarely assess cognition while language testing is generally very limited, or nonexistent, in investigations of people with amnesia (Hinckley, 2002). As noted above, previous studies of errorless learning suggest that memory and feedback can be important determinants of therapy outcome. It is important, therefore, to assess the more general cognitive abilities of people with aphasia and to relate these to therapy outcomes. It would have been interesting, therefore, to investigate the possibility that concurrent cognitive impairment, e.g. amnesia or executive-attentional deficits, influences the efficacy of errorful and errorless interventions. Unfortunately, too few studies reported sufficient background neuropsychology to be certain about the status of these cognitive systems in people with aphasia.

The review of the anomia treatment literature highlighted a reasonable number of studies that can be classified as error reducing. Although the number of therapies utilizing errorful techniques outweighed those with some form of errorless learning, the rate of success was equivalent for both types of therapy. Although the number of studies prevented formal statistical analysis, the review found evidence to suggest that errorless approaches are just as likely to achieve a positive outcome (in terms of immediate effect, follow-up testing, and generalization). This did not seem to vary if the studies 
were split by therapy type, principal impairment, or patient type. There were, however, a number of areas where there was very little information. For example, there were very few examples of errorless learning for treating anomia with a receptive technique. Likewise, there was only one study that used an error-reducing approach with a nonfluent patient. It is also notable that many errorless learning studies did not report long-term effects and generalization.

It is not surprising to find that only two therapies used the potentially optimal, error-eliminating approach. This may be because articles did not report error rate, or it may be that, in practice, it is extremely difficult to prevent people from making mistakes. The review showed that errorreducing techniques do have positive effects for people with word-finding difficulties. However, there is limited information on which to judge whether this technique is significantly advantageous over errorful approaches for treating word-finding difficulties - though the review did show that errorreducing techniques are at least as good as the more traditional trial-anderror interventions. In addition, there was very little information about the effects of error-reducing methods on untreated items and whether there was a lasting effect of training because very few studies included the necessary measures (Fillingham, Hodgson, Sage, \& Lambon Ralph, 2003).

\section{Novel therapy studies designed to compare errorless and errorful learning directly}

Word-finding difficulties are a key and frustrating symptom of Alzheimer's disease that typically follow the initial period of amnesia (Lambon Ralph, Patterson, Graham, Dawson, \& Hodges, 2003). Word-finding difficulties are also commonly found in people with aphasia after stroke (Lambon Ralph et al., 2002). A key aim of therapy/intervention is to tackle these wordfinding difficulties that otherwise cause frustration, embarrassment, and disability. We wanted to test the efficacy of errorless learning for aphasic word-finding difficulties and to begin to address some of the issues associated with the application and theory of errorless learning in this domain. Knowing which techniques are advantageous for which people would be extremely valuable knowledge for speech and language therapists (Nickels, 2002). We investigated the efficacy of errorless learning for the amelioration of anomia and directly contrasted this technique with errorful learning (trial and error), which is more traditionally used as a rehabilitation technique with people with aphasia. The specific research questions were as follows:

(1) Is errorless learning an effective therapy for anomia in people with aphasia? (Is it better than an equivalent intervention using traditional trial-and-error techniques?)

(2) Does the advantage of errorless learning depend on aphasia severity? 
(3) Does the effectiveness of errorless learning depend on neuropsychological severity?

(4) Does therapy generalize to untreated items?

(5) Does the advantage for errorless learning depend on the ability to use feedback?

(6) Is the positive effect of errorless learning dependent on the number of items in the training set?

To answer these questions, a sequence of three targeted therapy studies was carried out. The three therapy studies were designed to be linked to enable comparisons to be made across them. All participants were asked to complete the same battery of tests so that direct comparisons between participants could be made, and the severity of different underlying impairments could be related to therapy outcome. In order to maintain this link, the same participants were kept, and the core methodology was repeated, with some manipulation to enable specific research questions to be addressed. A case series design was used to facilitated this methodology (Lambon Ralph et al., 2002).

\section{Basic methodology}

A case series of people with aphasia with varying anomia severity, underlying cause of word-finding difficulty, and neuropsychological skill was used for each of the three studies. Not all of the participants from the study 1 were able to complete studies 2 and 3. Each participant was selected by criteria (see Fillingham, Sage, \& Lambon Ralph, 2005a) that allowed a wide variety of aphasia severity to be investigated. The participants' age ranged from 40 to 80 (mean 68), and the year of their cerebral vascular accident ranged from 1987 to 2001 (mean 1997). Each participant undertook a comprehensive battery of neuropsychological and aphasiological assessment. These revealed a phonological impairment in all cases except for SC. Formal assessment with semantic tasks revealed at least some comprehension/semantic problems in many of the cases too (with the exception of ME, HA, and GP). Some patients also had impaired comprehension. Interestingly, all patients were impaired on simple tests of divided attention and varied in their ability on classical tests of executive function (which turned out to be importantsee below). The methods used in the previous amnesia and anomia studies informed the nature of the therapies adopted such that they were representative of previous errorless learning techniques and were akin to clinical practice. An unelaborated method was used so that errorless and errorful techniques were directly comparable and efficacy could be judged against the language and neuropsychological measures for the case series of participants. Phonemic and orthographic cues were combined together with the picture so that all modalities were being used. The therapy tasks included sets of pictures that were matched for phoneme length, number of syllables, and 
frequency, within studies and across studies. In each of the studies, the two forms of intervention were run in a standard therapy procedure. The core methodology remained the same over the course of the three studies; however, there were small changes made. Table 9.1 shows the similarities and differences between the three studies.

Table 9.1 Summary of methodology for the three studies

\begin{tabular}{|c|c|c|c|}
\hline Study no. & $\begin{array}{l}1 \text { (Fillingham et al., } \\
2005 a)\end{array}$ & $\begin{array}{l}2 \text { (Fillingham, } \\
\text { Sage, Eु Lambon } \\
\text { Ralph, 2006) }\end{array}$ & $\begin{array}{l}3 \text { (Fillingham, Sage, } \\
\text { E⿱ Lambon Ralph, } \\
\text { 2005b) }\end{array}$ \\
\hline Participants & $\begin{array}{l}\text { HF, FO, RD, EW, } \\
\text { RR, JS, RH, ME, } \\
\text { HA, GP, SC }\end{array}$ & $\begin{array}{l}\text { HF, RD, RR, JS, } \\
\text { ME, HA, SC }\end{array}$ & $\begin{array}{l}\text { HF, RR, JS, RH, } \\
\text { ME, HA, SC }\end{array}$ \\
\hline $\begin{array}{l}\text { No. items per } \\
\text { condition }\end{array}$ & 30 & 20 & 20 \\
\hline No. sessions & 10 & 10 & 10 \\
\hline Method & Consecutively & Concurrently & Concurrently \\
\hline $\begin{array}{l}\text { No. items per } \\
\text { session }\end{array}$ & 30 & 40 & 40 \\
\hline Therapy* & $\begin{array}{l}\text { EL_read and/or } \\
\text { repeat } \\
\text { EF-progressive } \\
\text { phonological and } \\
\text { orthographic cueing }\end{array}$ & $\begin{array}{l}\text { EL_read and/or } \\
\text { repeat } \\
\text { EF-first } \\
\text { phoneme and } \\
\text { grapheme cue }\end{array}$ & $\begin{array}{l}\text { EL_read and/or } \\
\text { repeat } \\
\text { EF-first phoneme } \\
\text { and grapheme cue }\end{array}$ \\
\hline $\begin{array}{l}\text { No. attempts at } \\
\text { each item during } \\
\text { therapy }\end{array}$ & $\begin{array}{l}90 \text { - errorless } 120 \\
\text { (potentially)- } \\
\text { errorful }\end{array}$ & 30 & 90 \\
\hline Feedback & Yes & No & No \\
\hline
\end{tabular}

*EL: errorless learning; EF: errorful learning.

\section{Study 1}

In study 1 (Fillingham et al., 2005a), errorless therapy consisted of providing the patient with the picture along with its spoken and written name to ensure the correct response was made. The patient was told, "I am going to ask you to name the picture. First, I will tell you the name. Then I will show you the picture. The name will be written underneath the picture. We will do this three times." In errorful therapy, subjects were asked to name the picture, and if the response was incorrect, they were provided with progressive phonemic and orthographic cueing (Herbert, Best, Hickin, Howard, \& Osborne, 2001; Lambon Ralph et al., 2002; Lambon Ralph, Sage, \& Roberts, 2000). If the word was monosyllabic, the first phoneme and letter were given initially. If they were still unable to name the item, the first two phonemes 
and letters were given. If they were still incorrect, the whole word was given to repeat and/or read. If the word was multisyllabic, the first phoneme and letter were given, then the first syllable (spoken and written), and finally the whole word spoken and written (repeat/read). The 30 items were cycled through three times in each therapy session, which lasted 25-40 minutes. In study 1 , errorless learning proved to be as effective as the more traditional errorful learning (the raw scores were extremely similar for both methods across all patients: see Figure 9.1). The most striking finding was that language skill did not predict therapy outcome. Nor did any specific aspect of the patients' language profile (e.g., degree of phonological or semantic impairment). Instead, the participants' that responded better overall had better recognition memory, executive/problem-solving skills, and monitoring ability. It was concluded that these factors are essential cognitive components for providing effective monitoring and feedback systems to a more general learning mechanism. However, there were two main issues, one methodological and the other theoretical, which could have affected the results. First, the order of therapies was not counterbalanced across the participants. We chose to order the therapies so that the participants were given an initial period of therapy in which no errors occurred (errorless learning therapy was carried out first), to accustom them to this different form of therapy. This means, of course, that there might have been effects of this specific order on the results. Secondly, it was thought that feedback was available to the participants implicitly during errorful therapy (they knew when they had produced the correct answer because they moved onto the next item no matter what stage of the cueing process they were at). In other studies, the feedback reduced the underlying difference between the two, such that errorful learning produced equivalent results to errorless learning (McCandliss et al., 2002).

The aphasia literature contains very little information about the role of feedback. There appears to be no studies that have manipulated the use of feedback, or, if they have, it has not been the focus of such studies (Fink, Brecher, Schwartz, \& Robey, 2002). Even discussion of therapy practice gives contradictory advice about the utility of feedback. For example, Schuell's stimulation approach suggests that "Feedback about response accuracy should be provided when such feedback appears beneficial. The necessity for feedback may vary from patient to patient, but it generally is advisable" (Duffy, 1986, p. 150). However, it also argues: "It seems that confirmation of adequate performance may be helpful and encouraging and generally represents good clinical practice. Explanation and correction, on the other hand, should be carefully controlled and concise, bearing in mind that such feedback may be of little value, may waste time, and may be counterproductive" (Duffy, 1986, p. 165). Empirical studies for the role of feedback are clearly required. 


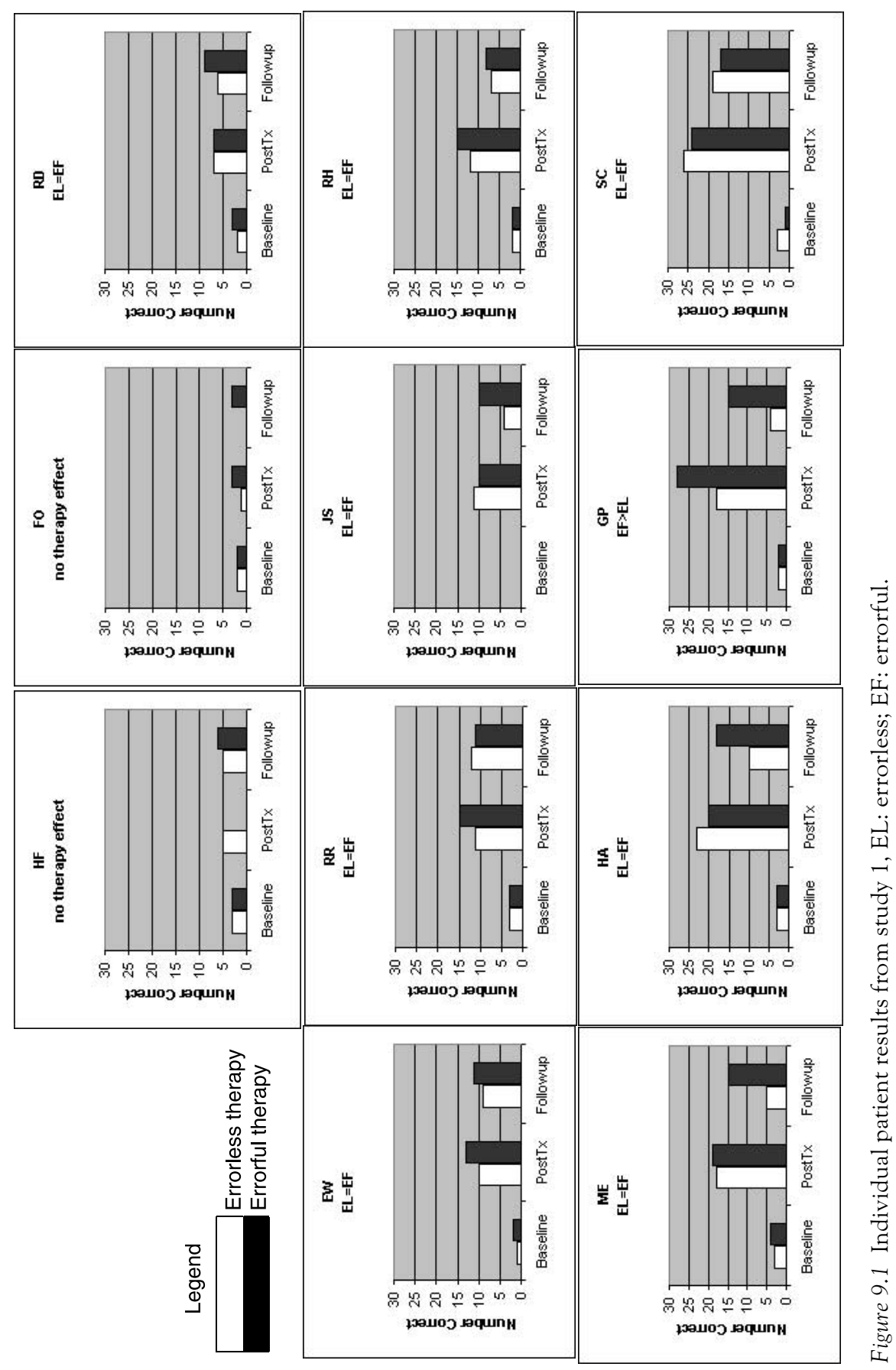




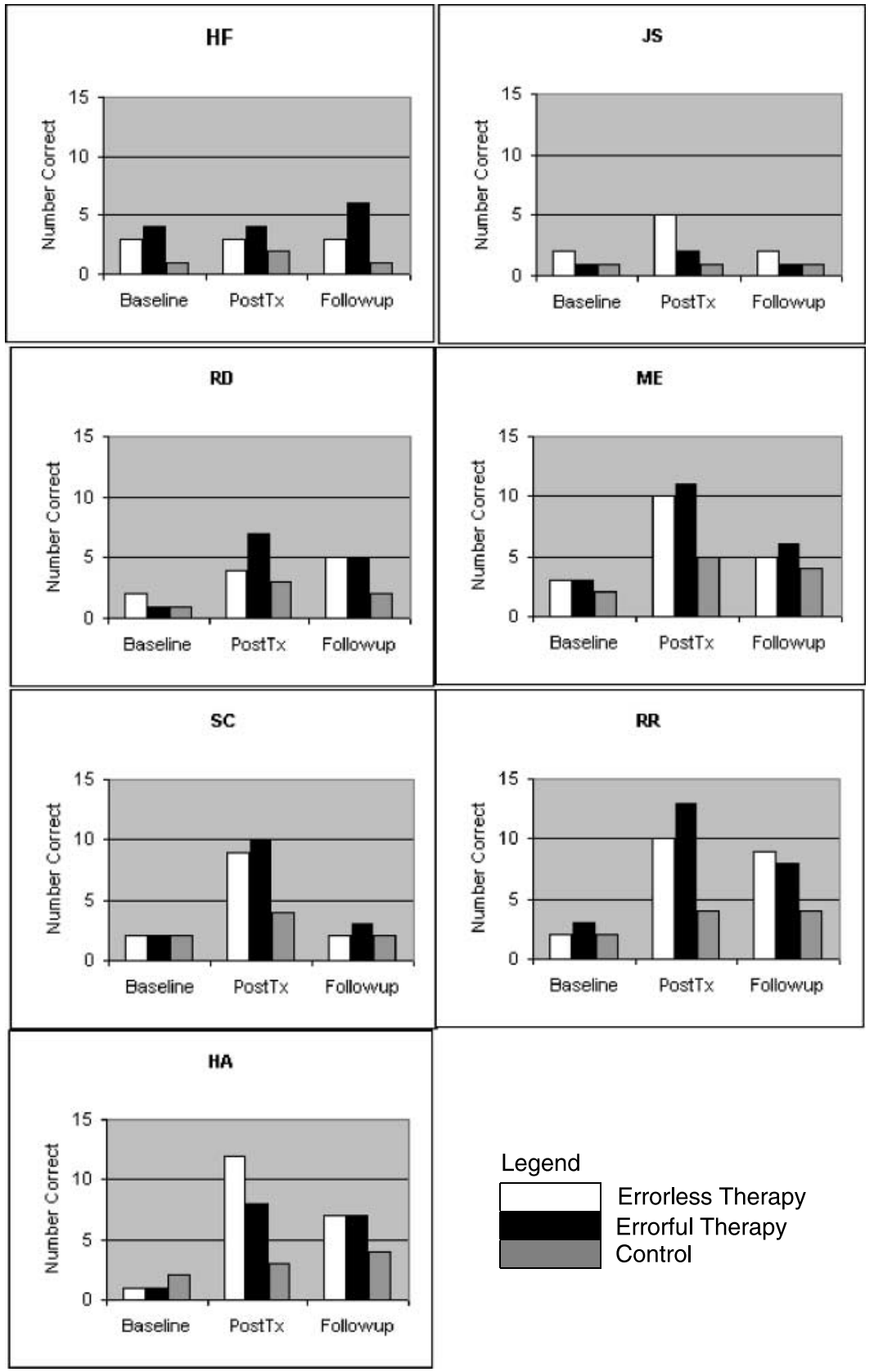

Figure 9.2 Individual patient results from study 2. 


\section{Study 2}

These potential confounds were addressed in study 2 (Fillingham, Sage, \& Lambon Ralph, 2005b). In this investigation, errorless and errorful learning therapies were given simultaneously and feedback was eliminated. Errorless therapy consisted of providing the participant with the picture along with its spoken and written name to ensure the correct response was made. The participant repeated and/or read the name once. In errorful therapy, the participant was given the picture along with the first phoneme and grapheme and asked to name the picture. For both therapies, whether the response was correct or not, no feedback was given, and the next item was attempted. The 20 items were cycled through three times in each therapy session, which lasted 25-40 minutes.

The pattern of results was unchanged; both therapies were equally effective after therapy and at follow-up (Figure 9.2). Therefore, there was no indication that the order adopted in the original study had any particular effect on the results. Again, executive/problem-solving skills and monitoring ability predicted immediate naming improvements, not language skill or specific aspects of the patients' language profile. Surprisingly, there was no effect of omitting feedback on the equivalence of each technique. It is thought that feedback is used to aid learning (Duffy, 1986); however, there are no empirical data for this patient group to support this view. However, in comparing the overall results immediately after treatment against the original study, it was clear that there was a reduction in the overall amount of learning in study 2. There were three methodological changes that could have contributed to this reduction in therapy effectiveness; feedback was omitted, the number of naming attempts per session for each item was reduced, and there was an increase the number of items for learning in each therapy session.

\section{Study 3}

Study 3 (Fillingham, Sage, \& Lambon Ralph et al., 2006 (Figure 9.3)) was carried out to replicate the previous findings, that errorless and errorful learning produce equivalent results and executive/problem-solving skills and monitoring ability predict therapy outcome, and to find out what contributed to the reduction in therapy from study 1 to study 2 . The methodology from study 2 was replicated with the exception that the number of naming attempts per session was increased to match the number of naming attempts in study 1. Errorless therapy consisted of providing the participant with the picture along with its spoken and written name to ensure the correct response was made. The participant repeated and/or read the name three times. In errorful therapy, the participant was given the picture along with the first phoneme and grapheme and asked to name the picture. This was done three times. For both therapies, whether the response was correct or not, 


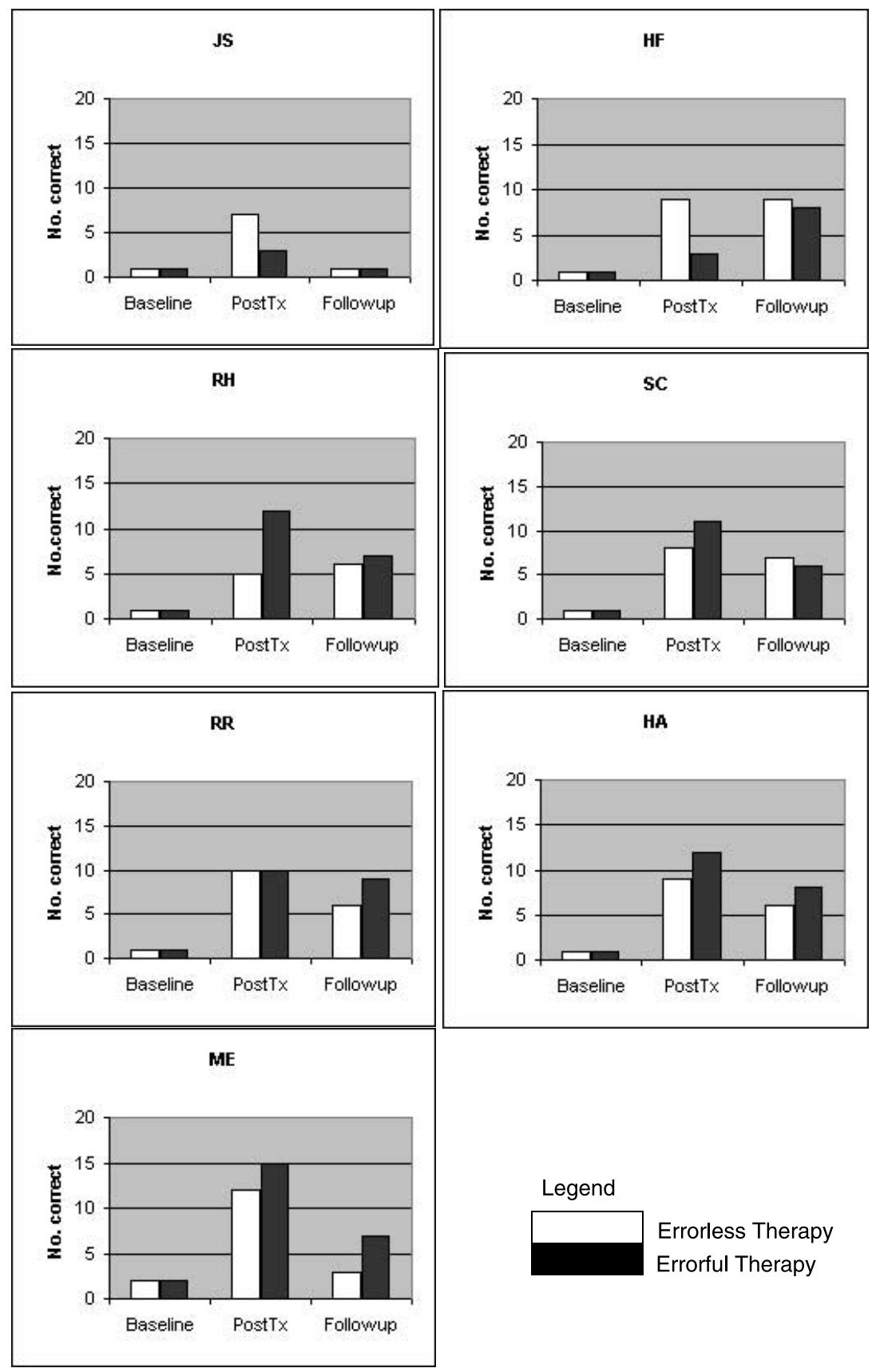

Figure 9.3 Individual patient results from study 3. 
no feedback was given and the next item was attempted. The 20 items were cycled through three times in each therapy session, which lasted 30-45 minutes. The results replicated the previous findings that errorless and errorful learning produce equivalent results immediately after treatment and at follow-up. Moreover, executive/problem-solving skills, monitoring ability, and recall memory predict immediate naming improvement, whereas the status of the participants' language skills do not.

When overall learning was compared over the three studies, two important findings emerged: (1) The greater the number of naming attempts at an item during therapy, the greater the success at learning the item; (2) giving or withdrawing feedback made no difference to therapy outcome. Figure 9.4 illustrates overall learning for all participants in each study.

\section{General discussion}

These findings pose a puzzle. Why are the two types of therapy equivalent for people with anomic aphasia, yet errorless learning appears to be better for people with memory impairment? The current literature does not contain a direct answer to this question. There are a number of variations between the amnesia studies and the anomia studies. The obvious differences between the two literatures are the diagnosis (aphasia/amnesia) and type of neurological disease (stroke/Alzheimer's disease). Another difference is the type of task. Wilson and colleagues have only ever directly compared the two types of learning in experimental tasks (e.g., stem completion); for therapeutic interventions, they have always used errorless learning alone. There are clear and potentially important differences between these tasks. During experimental studies involving stem completion, the participants are

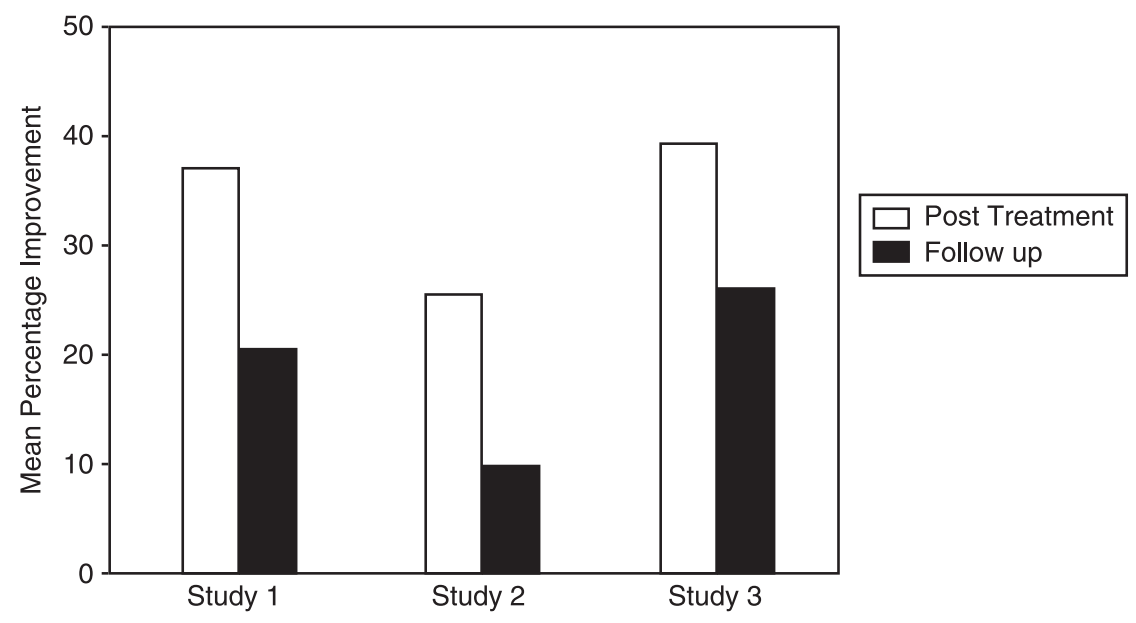

Figure 9.4 The mean percentage improvement for all participants, for each study, immediately after treatment and at follow-up. 
required to learn novel associations between fully formed, normal representations - that is, between the stem and a word in the person's existing vocabulary. During therapeutic intervention, the emphasis is on restoring damaged representations. One possibility, therefore, is that errorless learning is beneficial for some types of learning (e.g., novel associations) and not others (e.g., relearning representations). There is need for future research to address these issues by crossing the factors: people with aphasic anomia and people with Alzheimer's disease versus experimental tasks and therapeutic intervention. Because there are no empirical data to answer such questions, it will be assumed, for now, that experimental tasks and therapeutic tasks are tapping into the same learning/relearning system.

If this is the case, then the overall findings from the amnesia literature indicate that errorless learning produces superior performance to errorful learning. Currently, there are two explanations arising from the literature as to why this may be. The first, proposed initially by Wilson and colleagues, is one of a memory deficit (Baddeley \& Wilson, 1994). They proposed that errorless learning was effective as it capitalized on the intact implicit memory skills of people with amnesia. This has been debated by Squires et al. (1997), who argue that errorless learning profits from the residual explicit memory of people with amnesia. The second explanation is one of a source-monitoring difficulty - the patients are unable to determine whether the correct answer was their own self-generated incorrect guess or the experimenter's correction (O'Carroll et al., 1999).

However, errorless learning is advantageous for people other than those with memory impairment. As noted above, a recent study of learning in a normal adult system found that Japanese adults, who find it difficult to learn the distinction between the phonemes / / and / $\mathrm{r} /$ even after years of exposure to the English language, could learn the distinction if they were taught by an errorless technique. They also found that if feedback was introduced into the errorful paradigm, the adults would learn the distinction equally well in both errorless and errorful learning. McCandliss et al. (2002) concluded, therefore, that a model based solely on Hebbian learning (like McClelland et al., 1999) required an additional mechanism to capture the positive effects found in the feedback condition. Two possible mechanisms were proposed: Hebbian learning combined with error-correcting learning methods or Hebbian learning modulated by outcome information, as in reinforcement learning. This evidence indicates that memory impairments do not provide a complete explanation for the whole literature, as there are situations in which neurologically intact subjects benefit from an errorless learning technique.

The results from the three case-series studies presented here produced consistent findings. Although the participants varied greatly in the degree and type of aphasia, the two therapies produced equivalent results immediately after treatment, at follow-up, and for generalization. However, if a clinical judgement had to be made about which technique to use, then it was important to take into account the fact that all participants reported 
errorless learning as the preferable technique. Fillingham et al. (2003) noted that errorless learning is inherently monotonous and might suffer from being a passive treatment. Our experience suggests that participants were as likely to engage in the errorless learning therapy as any other. Indeed, the participants strongly preferred errorless treatment, as they found it less frustrating and more rewarding. This is probably because the errorless learning technique removes the need for people with aphasia, with sometimes severe expressive difficulties, to provide a spoken name repeatedly through each therapy trial. In this study, the participants were not bored by errorless therapy. There is, however, a danger that it may be inherently tedious for the therapist. If the positive results of errorless learning found here are replicated in future studies, alternative methods such as computerassisted therapy may prove to be the most effective way to deliver this type of intervention (see Fink et al., 2002, for an example). Another finding was that the outcome of immediate naming improvement can be predicted by the status of the participants' cognitive skills (executive/problem solving, monitoring ability, and memory), not language skills per se. Perhaps the most striking example of the lack of a relationship between language skills and therapy outcome was found in the results of participant JS. He was the most severe aphasic in our series (a global aphasic), yet he was in the middle of the range with respect to the size of his therapy effect. This interim position aligns closely with his performance on the Wisconsin Carding Sorting Test (Grant \& Berg, 1993) - an assessment that almost perfectly ranks the participants for therapy outcome (see Fillingham et al., 2005a). This result replicates a finding that has been shown previously for rehabilitation in other neurologically damaged patients, namely, that frontal executive systems are crucial for rehabilitation (Robertson \& Murre, 1999). Over the course of the three studies, we also found that a greater number of naming attempts at an item during therapy influenced learning success, while giving or withdrawing feedback makes no difference to therapy outcome.

\section{Toward a complete account and theoretical framework}

To devise a complete account, the following findings need to be taken into consideration:

(1) People with amnesia learn better with an errorless technique.

(2) Japanese adults, who are neurologically intact, also learn better with an errorless technique, but they can learn equally well when feedback is given in an errorful situation.

(3) People with aphasia, who have varying degrees of attentional-executive skills, either fail to learn with either technique or, for those that do learn, show an outcome that is equivalent for both techniques.

(4) The outcome of immediate naming improvement, for people with 


\section{Automaticity and Control in Language Processing}

aphasia, can be predicted by the status of attentional-executive skills and, to a lesser extent, memory.

(5) The number of naming attempts per session influences the outcome of therapy in people with aphasia.

(6) Feedback during an errorless and errorful technique, for people with aphasia, makes no difference to therapy outcome.

In an attempt to unify these various findings, we offer the following simple generic framework (Figure 9.5). The framework represents a single learning system made up of two component elements. The left-hand side (shown in boxes with roman font) represents a simplified information-processing framework in which input is transferred to output according to a series of internal representations. For example, during picture naming, there may be a series of representations involved, including visual, semantics, and phonology, all of which are required to drive speech production from a picture input.

The right-hand side of the model (depicted by lines and circles with italic



Figure 9.5 A framework for learning in errorless and errorful situations. 
font) involves components that adjust these internal representations. The adjustment of representations might be based on a Hebbian learning process (depicted as item 1 on Figure 9.5) that operates at the neural level in much the same way as the computational model of McClelland et al. (1999). This learning system is modulated by outcome (depicted as item 2 on Figure 9.5) through a series of linked components (shown as items $2 \mathrm{a}, 2 \mathrm{~b}$, and $2 \mathrm{c}$ on Figure 9.5). If this modulatory process is intact, learning should proceed and be equivalent for errorless and errorful learning. If any one of these components is compromised, then, unless learning stops altogether, errorless learning would be expected to be the superior method because it can rely solely upon Hebbian learning.

Modulation of learning probably requires the following elements. The initial stage is one of detection - that is, is the response correct or incorrect? The first part of this stage may simply be the detection of a difference between intended and observed outcome. If patients are unable to detect that, for example, their erroneous speech is not leading to the desired outcome, there may be no internal impetus for learning. This would seem to explain why patients with very poor attentional-executive skills show the poorest recovery (Robertson \& Murre, 1999), and why some of the people with aphasia that we studied did not improve with therapy. If patients can detect that something has gone awry with their response, learning is turned on in an attempt to adjust the internal representations.

For good learning in an errorful situation, the underlying Hebbian process needs to be gated appropriately. For this to happen, the response must be coded and distinguished from any alternatives, and the system must be able to distinguish correct responses from errors so that only the correct form is reinforced. These elements will be reliant on the status of internal representations (to distinguish correct from incorrect responses), memory, and attentional-executive mechanisms. Memory may be a key element in that, for learning to occur, patients may need to encode the stimulus, their response, and any therapist feedback. There are at least two ways that a gating process could operate (see McClelland et al., 1999). The first, much simpler version proposes that when a response is detected as correct, Hebbian learning continues to reinforce this correct stimulus-response pairing. If the response is detected as incorrect, learning is switched off. In this way, the system will gradually move toward an internal representation that is more likely to make a correct response and less likely to make an error. The second possible explanation involves an error-correcting learning mechanism that switches on when a trial leads to an erroneous response but continues with Hebbian learning to reinforce correct responses. These accounts need more investigation. Specifically, there is a need for a detailed computational model of learning to distinguish whether a simple gating system is enough for learning or whether the gating needs a mechanism for correcting errors. 


\section{Automaticity and Control in Language Processing}

\section{How might this generic framework account for the six key findings listed above?}

(1) The findings from the amnesia literature fit with this account in the following way. Unlike some of the participants in this study, the people with amnesia at some level know that they need to learn and/or that they are in a learning situation, so their learning system is switched on. However, their poor memory affects their ability to code and maintain representations for the stimulus and their response (see item $2 \mathrm{~b}$ on Figure 9.5). In this way, their poor memory skills disable the gating process, so that it is either functioning poorly or is dysfunctional. Consequently, the system is reliant on the basic Hebbian learning system - that is, learning will be better in an errorless situation.

(2) This account also explains why Japanese adults are poor at the auditory discrimination of $/ 1 /$ and $/ \mathrm{r} /$ even after years of exposure to English. Clearly, the Japanese adults know that they are failing, so their learning system is switched on. Their underlying representations for the receptive task cannot distinguish correct and incorrect responses (see item $2 \mathrm{~b}$ on Figure 9.5); therefore, the system has no basis for detecting errors. So even though they are not amnesic (they may have some memory for the auditory form and their response), they have no basis on which to gate learning. This means that they can use Hebbian learning, but they cannot engage effective modulation, as they do not know when to gate the system-much like the source-monitoring difficulty described by O'Carroll et al. (1999). Therefore, they-like the people with amnesia-benefit from errorless learning, but for a slightly different reason. Their difficulty can be overcome by replacing the gating with external feedback, such that they are able to learn in an errorful situation when they are told whether their response is correct or not.

(3) and (4) The people with aphasia we studied have a damaged internal representation. On the whole, they have intact memory skills but vary in attentional-executive ability. The participants with the poorest attentional-executive skills do not detect that anything is wrong (see item $2 \mathrm{a}$ on Figure 9.5) and therefore have no need to switch on their learning system. Consequently, they do not benefit from therapy by either errorless or errorful learning. The more attentional-executive skills people with aphasia have, the more likely they are to be able to realize that something is wrong. They are able to and do switch on their learning system. Given that these people with aphasia have at least a degree of attentional-executive abilities and, on the whole, good memory (see items $2 b$ and $2 c$ on Figure 9.5), they can engage all elements of the gating system, and thus they are able to learn in an errorful situation. This enables learning to occur equivalently in both an errorless and errorful situation.

(5) The account for the influence of the number of naming attempts and 
learning is relatively straightforward. Much like frequency effects in normal subjects, the more attempts at an item during learning, the more opportunity there is for the correct stimulus-response to be reinforced.

(6) The influence of experimenter/therapist feedback can be considered within this framework. For the Japanese adults, feedback effectively replaces the internal system(s) that would normally detect and distinguish errors from correct responses. In the people with aphasia, it seems possible that, apart from those participants who do not learn at all, the gating system is relatively intact and the therapist feedback is superfluous.

In conclusion, we believe that the combination of experimental neuropsychology with speech and language therapy provides a very productive partnership. In doing so, we have been able to bring insights from the neuropsychology of amnesia and contemporary findings from neuroscience to bear upon the treatment of a disabling and frustrating symptom of aphasia. In turn, the therapy studies have brought new information and insights not only specifically about the nature of relearning in the language domain but also more generally, about the plasticity of the human brain.

\section{Acknowledgement}

This work was supported by the Health Foundation (Award ref. no. 1727/1000).

\section{References}

Baddeley, A., \& Wilson, B. A. (1994). When implicit learning fails: Amnesia and the problem of error elimination. Neuropsychologia, 32, 53-68.

Baddeley, A. D. (1992). Implicit memory and errorless learning: A link between cognitive theory and neuropsychological rehabilitation? In L. R., Squire N. Butters (Eds.), Neuropsychology of memory (2nd ed.) (pp. 309-314). New York: Guilford Press.

Brown, P., Lupker, S. J., \& Colombo, L. (1994). Interacting sources of information in word naming: A study of individual differences. Journal of Experimental Psychology: Human Perception and Performance, 20, 537-554.

Brownjohn, M. D. (1988). Acquisition of Makaton symbols by a young man with severe learning difficulties. Behavioural Psychotherapy, 16, 85-94.

Buonomano, D. V., \& Merzenich, M. M. (1998). Cortical plasticity: From synapses to maps. Annual Review of Neuroscience, 21, 149-186.

Cipani, E., \& Spooner, F. (1997). Treating problem behaviors maintained by negative reinforcement. Research in Developmental Disabilities, 18, 329-342.

Clare, L., Wilson, B. A., Breen, K., \& Hodges, J. R. (1999). Errorless learning of face-name associations in early Alzheimer's disease. Neurocase, 5, 37-46.

Clare, L., Wilson, B. A., Carter, G., Breen, K., Gosses, A., \& Hodges, J. R. (2000). Intervening with everyday memory problems in dementia of Alzheimer type: An 


\section{Automaticity and Control in Language Processing}

errorless learning approach. Journal of Clinical and Experimental Neuropsychology, $22,132-146$.

Ducharme, J. M. (1996). Errorless compliance training: Optimizing clinical efficacy. Behavior Modification, 20, 259-280.

Ducharme, J. M., Atkinson, L., \& Poulton, L. (2000). Success-based, noncoercive treatment of oppositional behavior in children from violent homes. Journal of the American Academy of Child and Adolescent Psychiatry, 39, 995-1004.

Duffy, J. R. (1986). Schuell's stimulation approach to rehabilitation. In E. D. Chapey (Ed.), Language intervention strategies in adult aphasia (pp. 146-174). Baltimore, MD: Williams and Wilkins.

Duffy, L., \& Wishart, J. G. (1987). A comparison of two procedures for teaching discrimination skills to Down's syndrome and non-handicapped children. British Journal of Educational Psychology, 57, 265-278.

Duffy, L. A., \& Wishart, J. G. (1994). The stability and transferability of errorless learning in children with Down's syndrome. Down Syndrome: Research and Practice, 2, 51-58.

Evans, J. J., Wilson, B. A., Schuri, U., Andrade, J., Baddeley, A., Bruna, O., Canavan, T., Della Sala, S., Green, R., Laaksonen, R., Lorenzi, L., \& Taussik, I. (2000). A comparison of "errorless" and "trial-and-error" learning methods for teaching individuals with acquired memory deficits. Neuropsychological Rehabilitation, 10, 67-101.

Fillingham, J. K., Hodgson, C., Sage, K., \& Lambon Ralph, M. A. (2003). The application of errorless learning to aphasic disorders: A review of theory and practice. Neuropsychological Rehabilitation, 13, 337-363.

Fillingham, J. K., Sage, K., \& Lambon Ralph, M. A. (2005a). Further explorations and an overview of errorless and errorful therapy for anomia: The number of naming attempts during therapy effects outcome. Aphasiology, 19, 597-614.

Fillingham, J. K., Sage, K., \& Lambon Ralph, M. A. (2005b). The treatment of anomia using errorless learning vs. errorful learning: Are frontal executive skills and feedback important? International Journal of Language and Communication Disorders, 40, 505-524.

Fillingham, J. K., Sage, K., \& Lambon Ralph, M. A. (2006). The treatment of anomia using errorless learning. Neuropsychological Rehabilitation, 16, 129-154.

Fink, R. B., Brecher, A., Schwartz, M. F., \& Robey, R. R. (2002). A computerimplemented protocol for treatment of naming disorders: Evaluation of clinicianguided and partially self-guided instruction. Aphasiology, 16, 1061-1086.

Grant, D. A., \& Berg, E. A. (1993). Wisconsin Card Sorting Test. Odessa, FL: Psychological Assessment Resources, Inc.

Helm-Estabrooks, N. (2002). Cognition and aphasia: a discussion and a study. Journal of Communication Disorders, 35, 171-186.

Herbert, R., Best, W., Hickin, J., Howard, D., \& Osborne, F. (2001). Phonological and orthographic approaches to the treatment of word retrieval in aphasia. International Journal of Language and Communication Disorders, 36, 7-12.

Hinckley, J. (2002). Models of language rehabilitation. In P. Eslinger (Ed.), Neuropsychological interventions: Clinical research and practice (pp. 182-221). New York: Guilford Press.

Howard, D., Patterson, K., Franklin, S., Orchard Lisle, V., \& Morton, J. (1985). Treatment of word retrieval deficits in aphasia-a comparison of 2 therapy methods. Brain, 108, 817-829. 
Hunkin, N., Squires, E., Parkin, A., \& Tidy, J. (1998). Are the benefits of errorless learning dependent on implicit memory? Neuropsychologia, 36, 25-36.

Hunkin, N. M., Squires, E. J., Aldrich, F. K., \& Parkin, A. J. (1998). Errorless learning and the acquisition of word processing skills. Neuropsychological Rehabilitation, 8, 433-449.

Jackson, T. (1999). Dyspraxia: Guidelines for intervention. British Journal of Occupational Therapy, 62, 321-326.

Kern, R. (1996). Cognitive rehabilitation of people with mental illness. Psychiatric Rehabilitation Skills, 1, 65-73.

Lambon Ralph, M. A., Moriarty, L., \& Sage, K. (2002). Anomia is simply a reflection of semantic and phonological impairments: Evidence from a case-series study. Aphasiology, 16, 56-82.

Lambon Ralph, M. A., Patterson, K., Graham, N., Dawson, K., \& Hodges, J. R. (2003). Homogeneity and heterogeneity in mild cognitive impairment and Alzheimer's disease: a cross-sectional and longitudinal study of 55 cases. Brain, 126, 2350-2362.

Lambon Ralph, M. A., Sage, K., \& Roberts, J. (2000). Classical anomia: A neuropsychological perspective on speech production. Neuropsychologia, 38, 186-202.

Maxwell, J. P., Masters, R. S. W., Kerr, E., \& Weedon, E. (2001). The implicit benefit of learning without errors. Quarterly Journal of Experimental Psychology, 54, 1049-1068.

McCandliss, B., Fiez, J., Protopapas, A., Conway, M., \& McClelland, J. (2002). Success and failure in teaching the $/ \mathrm{r} /-/ 1 /$ contrast to Japanese adults: Tests of a Hebbian model of plasticity and stabilization in spoken language perception. Cognitive, Affective, and Behavioural Neuroscience, 2, 89-108.

McClelland, J., Thomas, A. G., McCandliss, B., \& Fiez, J. (1999). Understanding failures of learning: Hebbian learning, competition for representational space, and some preliminary data. Progress in Brain Research, 121, 75-80.

Merzenich, M., Jenkins, W., Johnston, P., Schreiner, C., Miller, S., \& Tallal, P. (1996). Temporal processing deficits of language-learning impaired children ameliorated by training. Science, 271, 77-84.

Morris, J., Franklin, S., Ellis, A. W., Turner, J. E., \& Bailey, P. J. (1996). Remediating a speech perception deficit in an aphasic patient. Aphasiology, 10, 137-158.

Nickels, L. (2002). Therapy for naming disorders: Revisiting, revising, and reviewing. Aphasiology, 16, 935-979.

O'Carroll, R. E., Russell, H. H., Lawrie, S. M., \& Johnstone, E. C. (1999). Errorless learning and the cognitive rehabilitation of memory-impaired schizophrenic patients. Psychological Medicine, 29, 105-112.

Parkin, A. J., Hunkin, N. M., \& Squires, E. J. (1998). Unlearning John Major: The use of errorless learning in the reacquisition of proper names following herpes simplex encephalitis. Cognitive Neuropsychology, 15, 361-375.

Robertson, I. H., \& Murre, J. M. J. (1999). Rehabilitation of brain damage: Brain plasticity and principles of guided recovery. Psychological Bulletin, 125, 544-575.

Squires, E. J., Hunkin, N. M., \& Parkin, A. J. (1997). Errorless learning of novel associations in amnesia. Neuropsychologia, 35, 1103-1111.

Tallal, P., Merzenich, M., Miller, S., \& Jenkins, W. (1998). Language learning impairments: integrating basic science, technology, and remediation. Experimental Brain Research, 123, 210-219.

Terrace, H. S. (1963). Discrimination learning with and without "errors". Journal of the Experimental Analysis of Behavior, 6, 1-27. 


\section{Automaticity and Control in Language Processing}

Wilson, B., \& Evans, J. (1996). Error-free learning in the rehabilitation of people with memory impairments. Journal of Head Trauma Rehabilitation, 11, 54-64.

Wilson, B. A., Baddeley, A., Evans, J., \& Shiel, A. (1994). Errorless learning in the rehabilitation of memory impaired people. Neuropsychological Rehabilitation, 4, 307-326.

Wykes, T., Reeder, C., Corner, J., Williams, C., \& Everitt, B. (1999). The effects of neurocognitive remediation on executive processing in patients with schizophrenia. Schizophrenia Bulletin, 25, 291-307. 